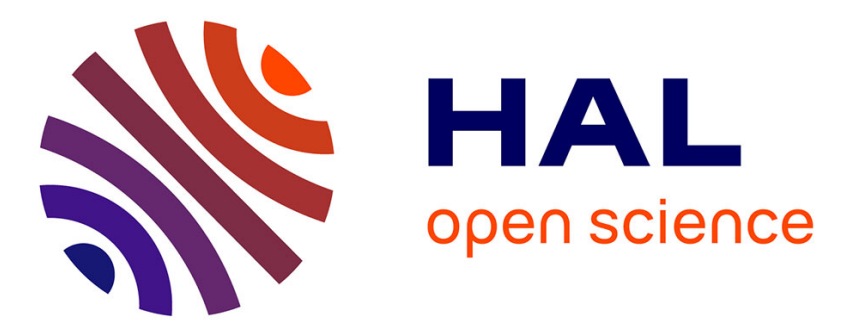

\title{
Tight Focusing Properties of Radially Polarized Doughnut Gaussian Beam through a Dielectric Interface
}

\author{
K. Prabakaran, R. Loganathan, A. Kesavan, K. Rajesh, A. Mohamed
}

Musthafa, V. Aroulmoji

\section{- To cite this version:}

K. Prabakaran, R. Loganathan, A. Kesavan, K. Rajesh, A. Mohamed Musthafa, et al.. Tight Focusing Properties of Radially Polarized Doughnut Gaussian Beam through a Dielectric Interface. International journal of advanced Science and Engineering, 2018, 5 (2), pp.896. 10.29294/IJASE.5.2.2018.896-900 . hal-03093631

\section{HAL Id: hal-03093631 \\ https://hal.science/hal-03093631}

Submitted on 26 Jan 2021

HAL is a multi-disciplinary open access archive for the deposit and dissemination of scientific research documents, whether they are published or not. The documents may come from teaching and research institutions in France or abroad, or from public or private research centers.
L'archive ouverte pluridisciplinaire HAL, est destinée au dépôt et à la diffusion de documents scientifiques de niveau recherche, publiés ou non, émanant des établissements d'enseignement et de recherche français ou étrangers, des laboratoires publics ou privés. 


\title{
Tight Focusing Properties of Radially Polarized Doughnut Gaussian Beam through a Dielectric Interface

\author{
K. Prabakaran ${ }^{*}$, R. Loganathan', A. Kesavan' ${ }^{1}$, K.B. Rajesh ${ }^{2}$, A. Mohamed Musthafa ${ }^{3}$, \\ V. Aroulmoji ${ }^{4}$ \\ ${ }^{1}$ Department of Physics, Mahendra Arts and Science College (Autonomous), Namakkal,Tamilnadu, India \\ 2Department of Physics, Chikkanna Government Arts College, Trippur, Tamilnadu, India \\ ${ }^{3}$ Department of General Studies (Physics Group), Jubail University College (Male Branch), \\ Royal Commission of Jubail, Kingdom of Saudi Arabia
} \\ ${ }^{4}$ Center for Research and Development, Mahendra Engineering College, Namakkal District, Tamil Nadu, India
}

\begin{abstract}
Tight focusing properties of an radially polarized doughnut Gaussian beam through a dielectric interface is theoretically investigated by vector diffraction theory. For the incident beam with topological charges $(m)$, probe depth $(d)$ and revive in the vicinity of focal plane, which results in the generation of many novel focal patterns. Such kind of focal structures may find applications in micro-particle trapping, manipulation, and material processing.
\end{abstract}

KEYWORDS: Radially polarized DG beam, Focal shift, Dielectric interface, Vector diffraction theory,

\section{INTRODUCTION}

In recent years, cylindrically polarized beam have attracted great interests because of its interesting properties and potential applications [1-4]. For example, tight focusing of radially polarized light beams can create a very strong longitudinal electric field component in the focal region [1]. Moreover, in many practical applications, an objective is used to focus an incident light beam through an interface between different media of different refractive indices. For example, in the application of semiconductor inspection, light beams are focused from air onto silicon substrate. Torok et al [5] developed the theoretical method for studying the focusing of an electromagnetic wave through dielectric interfaces [6, 7]. More recently, a sub-wavelength light needle with a longer depth of focus (over 9.5 $\lambda$ ) has been obtained using dual-beam focusing [8]. All these researches, the incident light beam is focused mainly in the vacuum. However, in some practical applications, the incident light beam is focused through an interface between different media by a high-NA lens. For example, in the case of optical trapping, a laser beam is focused through an interface between glass and water [9].

In recent times, many other kinds of beams, such as the Laguerre- Gaussian (LG) beam, the high order LG beam, the high order BG beam and the sinh-Gaussian beam, Multi gaussian beam, Hollow gaussian beam are discussed in depth [10-14]. Generally, their results have indicated that the form of the designed filters and their corresponding focusing performance are strongly influenced by the incident beam. Therefore, a new kind of radially polarized beam called doughnut Gaussian (DG) beam is introduced in a high NA focusing system. The defined DG beam is similar to a hollow Gaussian beam. In 2010, a subwavelength focal spot was achieved by using a radially polarized narrowwidth annular beam [15]. The DG beam, which is similar to the narrow-width annular beam, is falling to the category of the Gaussian beams [16].Considering that the intensity is null at the center of the doughnut beam, the focusing field of a radially polarized DG incident beam through a high NA lens will exhibit high resolution. Meanwhile, other focusing patterns, such as a doughnut beam and the special polarized beam, were also investigated, based on the filtering technology [17-21]. In this Letter, we study the tight focusing of radially polarized doughnut gaussian beam passing through dielectric interface by vector diffraction theory. The principle of the optical focusing radially polarized DG beam is given and the simulation results are discussed in depth.

\section{The principle of the optical focusing radially polarized DG beam}

The scheme of the optical system is shown in Fig. 1. Assume the interface between two dielectric media of refractive indices $n_{1}=1$ and $n_{2}=3.55$, such as focusing in air onto silicon substrate in the application of semiconductor inspection. The geometric focus of the objective without the interface is located at the origin of the coordinate system. The distance between the interface and the geometric focus is $d$ which is referred to as probe depth in Ref. [6]. For radially polarized beams, on the basis of vectorial Debye theory [2] the Cartesian components of the electric field vector in the focal region may be expressed as [22] 


$$
\begin{aligned}
& E(r, \varphi, z)=\left[\begin{array}{c}
E_{x}(r, \varphi, z) \\
E_{y}(r, \varphi, z) \\
E_{z}(r, \varphi, z)
\end{array}\right]=\frac{-i E_{0}}{\pi} \int_{0}^{\alpha} \int_{0}^{2 \pi} \exp \left[-i k_{0} \Phi\left(\theta_{1}, \theta_{2}\right)\right] \times \sin \theta_{1} \sqrt{\cos \theta_{1}} A\left(\theta_{1}\right) \\
& \times t_{p}\left[i k_{2} z \cos \theta_{2}+i k_{1} \sin \theta_{1} \cos (\varphi-\phi)\right]\left[\begin{array}{l}
\cos \theta_{2} \cos \varphi \\
\cos \theta_{2} \sin \varphi \\
\sin \theta_{2}
\end{array}\right] d \phi d \theta_{1} \rightarrow(1)
\end{aligned}
$$

Where $k_{i}=n_{i} k_{0}$ and $\mathrm{k}_{0}=2 \pi / \lambda$ is the wave number, $J_{n}(x)$ is the Bessel function of the first kind of order $n, \alpha=\arcsin (\mathrm{NA})$ is the maximal angle determined by the NA of the objective; $t_{p}$ is the amplitude transmission coefficients for parallel polarization states, which is given by the Fresnel equations [23].

$$
\begin{gathered}
t_{p}=\frac{2 \sin \theta_{1} \cos \theta_{1}}{\sin \left(\theta_{1}+\theta_{2}\right) \cos \left(\theta_{1}+\theta_{2}\right)} \rightarrow(2) \\
\text { The function } \Phi(\theta 1, \theta 2) \text { is given by } \\
\Phi\left(\theta_{1}, \theta_{2}\right)=-d\left(n_{1} \cos \theta_{1}-n_{2} \cos \theta_{2}\right) \rightarrow(3)
\end{gathered}
$$

Representing the so-called aberration function caused by and $\theta_{2}$ are related by the well-known Snell law. The Eq. (1) the mismatch of the refractive indices $n_{1}$ and $n_{2}$. Here $\theta_{1}$ could be simplified to

$$
E(r, \psi, z)=\left[\begin{array}{c}
E_{x}(r, \psi, z) \\
E_{y}(r, \psi, z) \\
E_{z}(r, \psi, z)
\end{array}\right]=-i^{n+1} E_{0}\left[\begin{array}{l}
i\left(I_{n+1 e^{i \psi}}-I_{n-1 e^{-i \psi}}\right) \\
I_{n+1 e^{i \psi}}+I_{n-1 e^{-i \psi}} \\
2 I_{n}
\end{array}\right] e^{i m \psi} \rightarrow(4)
$$

$I_{n}(r, z)=\int_{0}^{\alpha} \exp \left[-i k_{0} \Phi\left(\theta_{1}, \theta_{2}\right)\right] A\left(\theta_{1}\right) \sqrt{\cos \theta_{1}} \times \sin \theta_{1} t_{p} \sin \theta_{2} J_{n}\left(k_{1} r \sin \theta_{1}\right) \times \exp \left(-i k_{2} z \cos \theta_{2}\right) d \theta_{1} \rightarrow(5)$

$I_{n \pm 1}(r, z)=\int_{0}^{\alpha} \exp \left[-i k_{0} \Phi\left(\theta_{1}, \theta_{2}\right)\right] A\left(\theta_{1}\right) \sqrt{\cos \theta_{1}} \times \sin \theta_{1} t_{p} \cos \theta_{2} J_{n \pm 1}\left(k_{1} r \sin \theta_{1}\right) \times \exp \left(-i k_{2} z \cos \theta_{2}\right) d \theta_{1} \rightarrow(6)$

With similar simplification for the radially polarize doughnut gaussian beams can be written us

$$
E(r, \psi, z)=\left[\begin{array}{c}
E_{x}(r, \psi, z) \\
E_{y}(r, \psi, z) \\
E_{z}(r, \psi, z)
\end{array}\right]=i^{n+1} E_{0}\left[\begin{array}{l}
I_{n+1 e^{i \psi}}^{\prime}-I_{n-1 e^{-i \psi}}^{\prime} \\
-i\left(I_{n+1 e^{\prime}}^{\prime}+I_{n-1 e^{-i \psi}}^{\prime}\right) \\
0
\end{array}\right] e^{i m \psi} \rightarrow(7)
$$

where

$I_{n \pm 1}^{\prime}(r, z)=\int_{0}^{\alpha} \exp \left[-i k_{0} \Phi\left(\theta_{1}, \theta_{2}\right)\right] A\left(\theta_{1}\right) \sqrt{\cos \theta_{1}} \times \sin \theta_{1} t_{p} \cos \theta_{2} J_{n \pm 1}\left(k_{1} r \sin \theta_{1}\right) \times \exp \left(-i k_{2} z \cos \theta_{2}\right) d \theta_{1} \rightarrow(8)$

$A\left(\theta_{1}\right)$ describes the doughnut Gaussian beam, this function is given by $[24,25]$

\section{Prabakaran et al.,}




$$
A\left(\theta_{1}\right)=\exp \left[-\left(\frac{\sin (\theta)-\theta_{0}}{w_{0}}\right)^{2}\right] \rightarrow(9)
$$

Where $\omega_{0}$ reflects the beam size at the beam waist of the Gaussian beam. $\theta_{0}$ relates with the radius of the DG beam. $\theta$ is the variable of the function. Obviously, the shape of the defined doughnut Gaussian beam is determined by $\theta_{0}$ and $\omega_{0}$. To be more specific, the position of the maximum field intensity depends on $\theta_{0}$. For $\theta_{0}=0$ the beam governed by Eq. (9) is a conventional Gaussian beam. The width of the DG beam is determined by $\omega_{0}$.

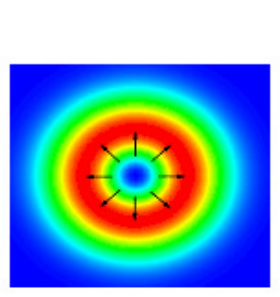

Radially Polarized DG beam

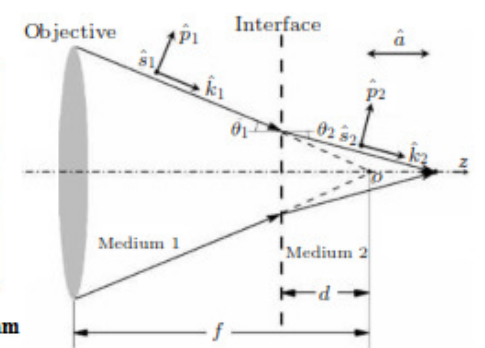

\section{RESULTS AND DISCUSSION}

Without loss of generality and validity, it is proposed that the parameter chosen as $\lambda=633 \mathrm{~nm}$. NA $=0.9, \theta_{0}=0.8$, and $w_{0}=0.125$. For all calculations in the length unit is normalized to $\lambda$ and the energy density is normalized to unity. Figure 2 illustrates the evolution of threedimensional light intensity distribution of high NA lens for the incident radially polarized doughnut Gaussian beam through dielectric interface.

Figure 2(a-c) shows the intensity distribution for topological charge $m=1$, probe depth $\mathrm{d}=1 \lambda$. It is observed from the figure due to the mismatch of refractive index across the interface, the on axial maximum intensity is not at the focus and it is shifted to $5 \lambda$ and is shown in Fig. 2 (a $\& \mathrm{c})$. The calculated focal depth is $8 \lambda$. The FWHM of the generated focal spot is $0.44 \lambda$ and is shown in Fig. 2(b). Moreover, it is observed from the figure that the longitudinal component is much smaller than the radial component. However, for $d=2 \lambda$, Its noted from Fig.2.(d), that the presence of dielectric interface shifts the position of an axial maximum intensity to $10 \lambda$ and the focal spot size is slightly reduced to $0.42 \lambda$, focal depth is improved to $11 \lambda$ and is shown in Figs. 2(e) and 2(f).

Fig.1.Schematic diagram of the optical system
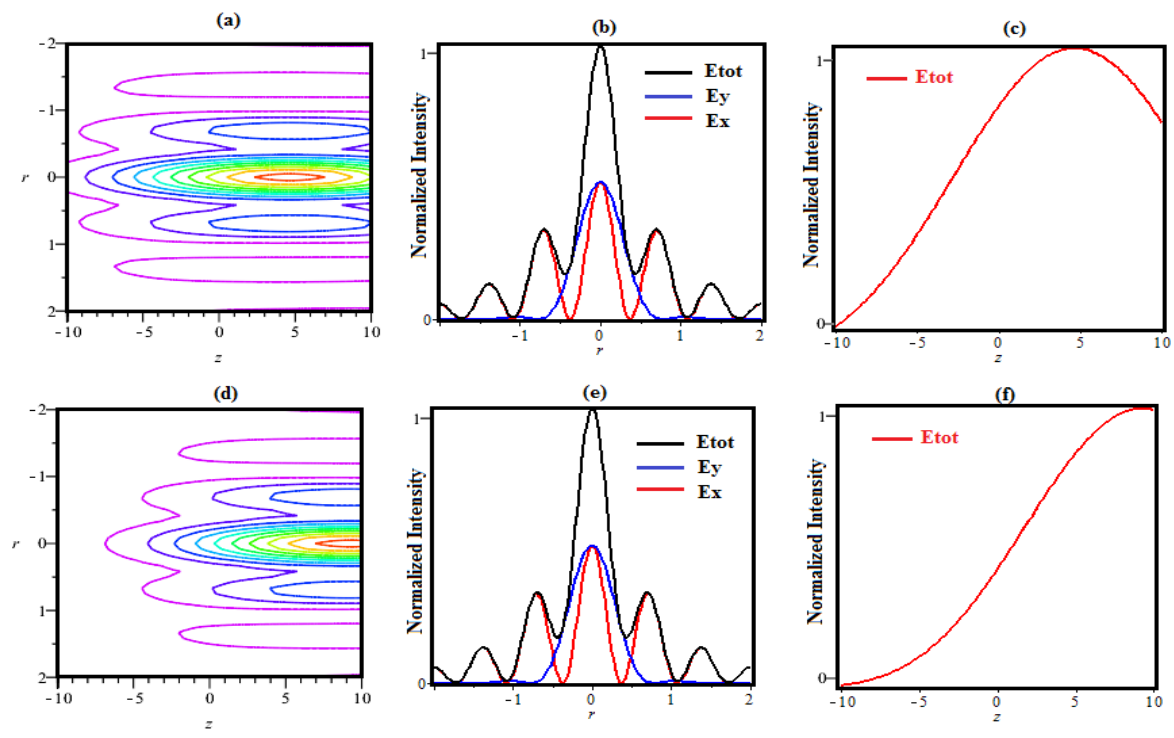

Fig.2. (a) 3d intensity distribution for topological charge $m=1$ and $d=1 \lambda$. (b) $2 d$ intensity of the components Ex (red line), Ey (blue line) and Etot (black line) at $\mathrm{z}=5 \lambda$. (c) On axial intensity at $r=0$. (d) $3 d$ intensity distribution for topological charge $m=1$ and $d=2 \lambda$. (e) $2 d$ intensity of the components $\operatorname{Ex}($ red line), Ey (blue line), and Etot (black line)at $\mathrm{z}=10 \lambda$ for $d=2 \lambda$.(f) On axial intensity at $r=0$.

\section{Prabakaran et al.,}



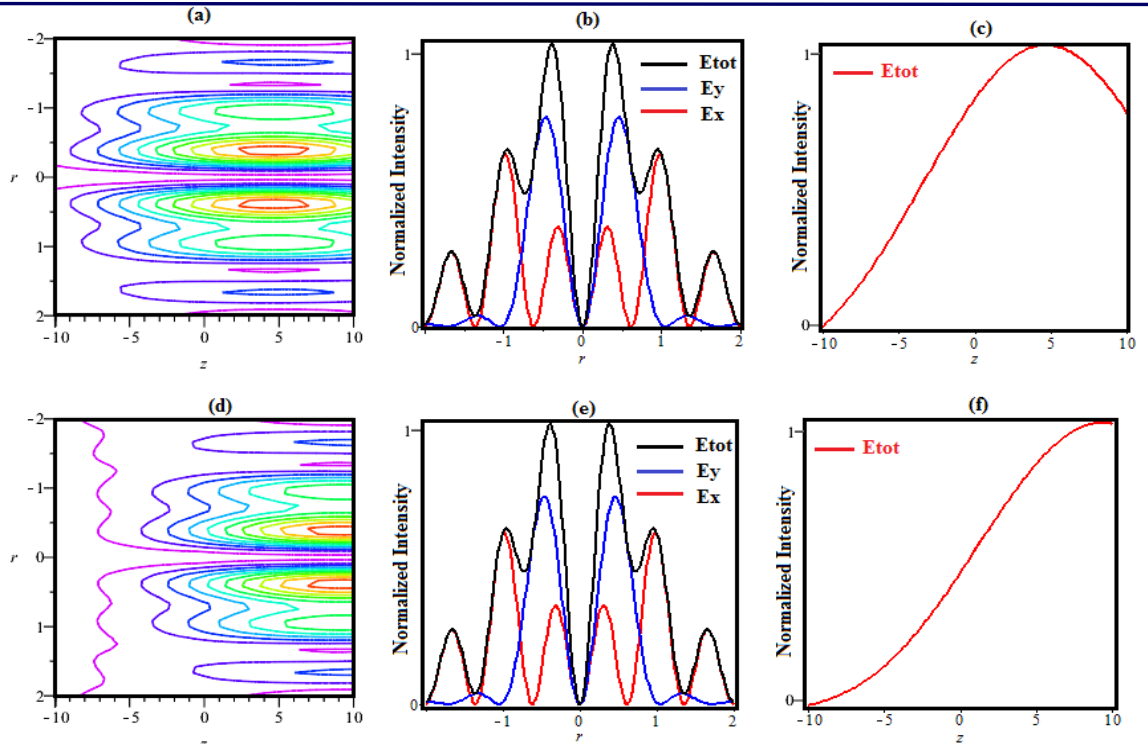

Fig.3. (a) 3d intensity distribution for topological charge $m=2$ and $d=1 \lambda$. (b) $2 d$ intensity of the components Ex (red line), Ey (blue line) and Etot (black line) at $\mathrm{z}=5 \lambda$. (c) $0 \mathrm{n}$ axial intensity at $\mathrm{r}=2 \lambda$. (d) $3 d$ intensity distribution for topological charge $m=2$ and $d=2 \lambda$. (e) $2 d$ intensity of the components $\operatorname{Ex}($ red line), Ey (blue line), and Etot (black line)at $\mathrm{z}=10 \lambda$ for $\mathrm{d}=2 \lambda$.(f) 0 n axial intensity at $\mathrm{r}=2 \lambda$.

Such a dark channel may have many applications such as imaging the silicon integrated circuit. We also observed the focal properties of the radially polarized doughnut Gaussian beam with the probe depth $d=1 \lambda$, topological charge $m=2$, the generated focal segment is a focal hole and shown in Fig. 3. It is observed from the figure the on axial maximum intensity is not at the focus and it is shifted to $5 \lambda$ as shown in Fig. 3(a). The calculated focal depth is $8.4 \lambda$ as shown in fig. 3 (c). The FWHM of the generated focal hole size is $0.34 \lambda$ as shown in Fig. 3 (b). Moreover, it is observed from the figure the longitudinal component is much higher than the radial component. However, by setting $d=2 \lambda$, we observed from the Fig.2.(e), increase in size of the probe depth further shifted the on axial maximum intensity to $10 \lambda$ and focal hole size is slightly reduced to $0.32 \lambda$, focal depth is improved to $9.6 \lambda$ as shown in Fig. 3(e), 3(f) respectively. We also observed that by altering the probe depth of the dielectric interface alters the focal shift position and focal depth of the generated focal segment. In optical trapping system, it is usually deemed that the forces exerted on the particle in light field consist of two kinds of forces, one is the optical gradient force, which play a crucial role in constructing optical trap and its intensity is proportional to the optical intensity gradient; the other kind of force is scattering force, which usually has complex forms because this kind of force is related to the properties of the trapped particles, and whose intensity is proportional to the optical intensity [26]. Therefore, tunable optical intensity distribution in focal region means that the controllable optical trap may occur [27]. The intensity distribution in focal region of the radially polarized DG beam can be altered considerably by beam parameter $m$ and probe depth $d$, and many novel focal patterns can occur, which can be used to construct tunable optical traps.

\section{CONCLUSION}

In summary, we have derived the expressions for intensity distribution of tightly focused radially polarized doughnut gaussian beam passing through a dielectric interface have been investigated theoretically by the vector diffraction theory. It is observed that the presence of dielectric interface one can generate many novel focal patterns such as focal spot and focal hole suitable for micro particle trapping, manipulation and material processing. We also observed that by altering the probe depth of the dielectric interface alters the focal shift position and focal depth of the generated focal segment.

\section{REFERENCES}

[1] Dorn,R., Quabis,S., Leuchs,G, 2003. Sharper focus for a radially polarized light beam, Phys. Rev. Lett. 91, 233901.

[2] Youngworth,K.S., Brown, T.G, 2000. Focusing of high numerical aperture cylindrical-vector beams, Opt. Express 7, 77-87.

[3] Lin, H., Jia, B.H., Gu,M, 2011. Generation of an axially super resolved quasi-spherical focal spot using an amplitude modulated radially polarized beam, Opt. Lett. 36, 2471-2473.

[4] Q. Zhan and J. R. Leger, Focus shaping using cylindrical vector beams, Opt. Express 10, 324-331 (2002).

[5] Török, P., Varga, P., Laczik,Z., Booker, G.R, 1995. Electromagnetic diffraction of light focused through a planar interface between materials of mismatched refractive indices: an integral representation. J. Opt. Soc. Am. A 12, 325-332.

[6] Török, P., Varga,P., Booker, G.R, 1995. Electromagnetic diffraction of light focused through a plana interface

\section{Prabakaran et al.,}


between materials of mismatched refractive indices: structure of the electromagnetic field. J. Opt. Soc. Am. A 12, 2136-2144.

[7] Török, P., Varga,P., Konkol,A., Booker,G.R, 1995. Electromagnetic diffraction of light focused through a planar interface between materials of mismatched refractive indices: structure of the electromagnetic field. II J. Opt. Soc. Am. A 13, 2232-2238.

[8] Kuang,C., Hao,X., Liu,X., Wang,T., Ku,Y, 2011 Formation of sub-half-wavelength focal spot with ultra long depth of focus, Opt. Commun. 284, 1766.

[9] Biss.D.P., Brown, T.G, 2009. Cylindrical vector beam focusing through a dielectric interface, Opt. Express 9,490-497.

[10] Wang,H., Shi,L., Lukyanchuk,B., Sheppard,C.J.R., Chong, C.T. 2008. Creation of a needle of longitudinally polarized light in vacuum using binary optics. Nat Photonics 2, 501-505.

[11] Cagniot Emmanuel, Fromager Michael, Godin Thomas, Passilly Nicolas, AïtAmeur Kamel. 2011. Transverse super resolution technique involving rectified Laguerre-Gaussian $\mathrm{LG}_{0 \mathrm{p}}$ beams. J Opt Soc Am A 28, 1709-1715.

[12] Sun, Q.G., Zhou, K.Y., Fang, G.Y.,Zhang, G.Q., Liu, Z.J., Liu, S.T. 2012. Hollow sinh-Gaussian beams and their paraxial properties. Opt Express 20, 96829691.

[13] William Charles,J., Prabakaran,K., Parimaladevi,R., Rajesh,K.B.,.Pillai, T.V.S.2014. Tight focus of a azimuthally polarized and amplitude-modulated annular multi-gaussian beam, Optik 125, 3023-3026.

[14] Prabakaran,K., Rajesh,K.B., Anbarasan,P.M, 2014. Tight focusing of phase modulated radially polarized hollow Gaussian beam using complex phase filter, Optik 125, 6965-6968.

[15] Kitamura K., Sakai K., Noda S. 2010. Sub-wavelength focal spot with long depth of focus generated by radially polarized, narrow-width annular beam. Opt Express 18, 4518-4125.
[16] Liu Zhengjun, Zhao Haifa, Liu Jianlong, Lin Jie, Ahmad Muhammad A, Liu Shutian, 2007. Generation of hollow Gaussian beams by spatial filtering. Opt Lett. 32, 2076-2078.

[17] Liu,T., Tan, J., Liu, J., Lin, J, 2013.Creation of subwavelength light needle, equidistant multi-focus, and uniform light tunnel, J.Mod. Opt. 60, 378-381.

[18] Yuan, G. H., Wei, S. B., Yuan, X.C, 2011. Non diffracting transversally polarized beam, Opt. Lett. 36, 3479-3481.

[19] Khonina, S. N, 2013. Simple phase optical elements for narrowing of a focal spot in high-numericalaperture conditions, Opt. Eng. 52,091711.

[20] Reddy, S. G., Kumar, A., Prabhakar, S., Singh, R. P, 2013. Experimental generation of ring-shaped beams with random sources, Opt. Lett. 38, 44414444.

[21] Khonina,S.N.,Alferov, Karpeev, S. V, 2013. Strengthening the longitudinal component of the sharply focused electric field by means of higherorder laser beams, Opt. Lett. 38, 3223-3226.

[22] Gu. M, 2000 Advanced Optical Imaging Theory (Berlin:Springer)

[23] Born,M., Wolf, E, 1999 Principles of Optics 7th edn (Cambridge: Cambridge University Press)

[24] Lin,J., Chen, R., Yu, H., Jin,P., Cada,M., Ma,Y, 2014. Analysis of sub-wavelength focusing generated by radially polarized doughnut Gaussian beam, Opt. Laser Technol. 64, 242-246.

[25] Prabakaran,K., Rajseh K.B., ,Ramachandran.S, Arulmoji, V. 2018. Tight Focusing Properties of Phase Modulated Longitudinally Polarized Doughnut Gaussian Beam, Int. J. Adv. Sci. Eng.4, 651-655.

[26] Visscher,K., Brakenhoff,G.J, 1992. Theoretical study of optically induced forces on spherical particles in a single beam trap I: Rayleigh scatterers, Optik 89, 174-180.

[27] Gao, X., Fei, Z., Xu,W., Gan, F, 2004.Focus splitting induced by a pure phase-shifting apodizer, Opt. Commun. 239, 55-59.

\begin{abstract}
All (C) 2018 are reserved by International Journal of Advanced Science and Engineering. This Journal is licensed under a Creative Commons Attribution-Non Commercial-ShareAlike 3.0 Unported License.
\end{abstract}

\title{
Prabakaran et al.,
}

